\title{
Inverse association between insulin resistance and gait speed in nondiabetic older men: results from the U.S. National Health and Nutrition Examination Survey (NHANES) 1999-2002
}

\author{
Chen-Ko Kuo1,2, Lian-Yu Lin ${ }^{3}$, Yau-Hua Yu ${ }^{5,6,7}$, Kuan-Han Wu ${ }^{1,2}$ and Hsu-
} $\mathrm{Ko} \mathrm{KuO} * 3,4,8$

\begin{abstract}
Address: ${ }^{1}$ Department of Emergency Medicine, Chang-Gung Memorial Hospital-Kaohsiung Medical Center, No.123, Dapi Rd., Niaosong Township, Kaohsiung County 833, Taiwan, 2Department of Emergency Medicine, Chang Gung University College of Medicine, 259 Wen-Hwa 1st Road, Kwei-Shan Taoyuan 333, Taiwan, ${ }^{3}$ Department of Internal Medicine, National Taiwan University Hospital, 7 Chun-Shan South Road, Taipei 100, Taiwan, ${ }^{4}$ Department of Geriatrics and Gerontology, National Taiwan University Hospital, 7 Chun-Shan South Road, Taipei 100, Taiwan, ${ }^{5}$ School of Dentistry, National Yang Ming University, No.155, Sec. 2, Linong St., Beitou Dist., Taipei 112, Taiwan, ${ }^{6}$ Department of Dentistry, Taipei Veterans General Hospital, No. 201, Sec. 2, Shih-Pai Road, Taipei 112, Taiwan, ${ }^{7}$ Department of Medical Research and Development, Taipei Veterans General Hospital, No. 201, Sec. 2, Shih-Pai Road, Taipei 112, Taiwan and ${ }^{8}$ Division of Gerontology Research, Institute of Population Health Sciences, National Health Research Institutes, 35 Keyan Road, Zhunan, Miaoli County 350, Taiwan
\end{abstract}

Email: Chen-Ko Kuo - chenkokuo@yahoo.com.tw; Lian-Yu Lin - lin7010@ms1.nihet.net; Yau-Hua Yu - yauhuayu@gmail.com; KuanHan Wu - hayatowu1120@gmail.com; Hsu-Ko Kuo* - hsukokuo@yahoo.com

* Corresponding author

Published: 19 November 2009

BMC Geriatrics 2009, 9:49 doi:10.1186/147|-2318-9-49

This article is available from: http://www.biomedcentral.com/I47I-23/8/9/49

(C) 2009 Kuo et al; licensee BioMed Central Ltd.

This is an Open Access article distributed under the terms of the Creative Commons Attribution License (http://creativecommons.org/licenses/by/2.0), which permits unrestricted use, distribution, and reproduction in any medium, provided the original work is properly cited.
Received: 27 May 2009

Accepted: 19 November 2009

\begin{abstract}
Background: Recent studies have revealed the associations between insulin resistance (IR) and geriatric conditions such as frailty and cognitive impairment. However, little is known about the relation of IR to physical impairment and limitation in the aging process, eg. slow gait speed and poor muscle strength. The aim of this study is to determine the effect of IR in performance-based physical function, specifically gait speed and leg strength, among nondiabetic older adults.

Methods: Cross-sectional data were from the population-based National Health and Nutrition Examination Survey (1999-2002). A total of II68 nondiabetic adults ( $\geq 50$ years) with nonmissing values in fasting measures of insulin and glucose, habitual gait speed (HGS), and leg strength were analyzed. IR was assessed by homeostasis model assessment (HOMA-IR), whereas HGS and peak leg strength by the 20-foot timed walk test and an isokinetic dynamometer, respectively. We used multiple linear regression to examine the association between IR and performance-based physical function.

Results: IR was inversely associated with gait speed among the men. After adjusting demographics, body mass index, alcohol consumption, smoking status, chronic co-morbidities, and markers of nutrition and cardiovascular risk, each increment of I standard deviation in the HOMA-IR level was associated with a $0.04 \mathrm{~m} / \mathrm{sec}$ decrease $(p=0.003)$ in the HGS in men. We did not find such association among the women. The IR-HGS association was not changed after further adjustment of leg strength. Last, HOMA-IR was not demonstrated in association with peak leg strength.

Conclusion: IR is inversely associated with HGS among older men without diabetes. The results suggest that IR, an important indicator of gait function among men, could be further investigated as an intervenable target to prevent walking limitation.
\end{abstract}




\section{Background}

Recently, increasing research effort has been focused on the role of IR in age-related conditions or geriatric syndromes. The Italian InCHIANTI study, by examining community-dwelling elderly population, suggested that IR is associated with cognitive impairment as evidenced by poor performance in Trail Making Test and Mini-Mental State Examination $[1,2]$. By following 3141 communitydwelling adults for 10 years, Barzilay and colleagues from the Cardiovascular Health Study demonstrated that IR is associated with incident frailty [3], a composite outcome consisting of involuntary weight loss, exhaustion, low physical activity, slowness, and weakness. With life expectancy reaching its historic pinnacle, decline in physical performance and the resultant late-life disability have become common features in an aging society [4-9]. However, data examining the association between IR and physical function are relatively sparse. We hypothesized that IR is inversely associated with physical function in community-dwelling elders. We sought to test the hypotheses by analyzing data from the NHANES 1999-2002.

\section{Methods}

\section{Study Design and Population}

The NHANES, a population-based survey, used a stratified, multistage, and cluster sampling design to obtain a representative sample of the noninstitutionalized U.S. civilian population. NHANES consists of a detailed home interview and a health examination conducted in a mobile examination center (MEC). Datasets, Survey Operations Manuals, Consent Documents, and Brochures of the NHANES 1999-2002 are available on the NHANES website $[10,11]$.

A subsample of 2052 participants aged 50 years and older were randomly assigned to a morning session and had complete information of fasting plasma glucose and serum insulin. Individuals who had diabetes (physiciandiagnosed history, fasting plasma glucose $\geq 126 \mathrm{mg} / \mathrm{dL}$, or current use of diabetes medication; $\mathrm{n}=434$ ) or with missing values in the timed walking and isokinetic leg strength tests because of safety concerns (recent chest or abdominal surgery; heart attack in the past 6 weeks; brain aneurysm or stroke; current neck or back pain; difficulty in bending or straightening right knee; or right knee or right hip replacement) or any administrative, communicative, or technical problems $(n=450)$ were excluded from the analysis, leaving 1168 participants as the analytic sample. Among the final sample, median values of $\mathrm{C}$-reactive protein $(0.27 \mathrm{mg} / \mathrm{dL}, \mathrm{n}=1)$, vitamin $\mathrm{B}_{12}(465.5 \mathrm{pg} / \mathrm{mL}, \mathrm{n}=$ $2)$, folate $(15.1 \mathrm{ng} / \mathrm{mL}, \mathrm{n}=3)$, total cholesterol $(212 \mathrm{mg} /$ $\mathrm{dL}, \mathrm{n}=2)$, cotinine $(0.05 \mathrm{ng} / \mathrm{mL}, \mathrm{n}=24)$, as well as body mass index $(27.12 \mathrm{~kg} / \mathrm{m} 2, \mathrm{n}=12)$ were used to impute missing values for these variables, all of which were used in the analysis.

\section{Measures of IR}

A blood sample was collected following an overnight fast $(\geq 8 \mathrm{~h}$ ) in participants who were assigned to a physical examination during a morning session. Plasma glucose level was measured with a hexokinase enzymatic reference method (COBRAS MIRA; Roche Diagnostics, Indianapolis, IN) and serum insulin level by a radio-immunoassay (Pharmacia Diagnostics, Uppsala, Sweden). Homeostasis model assessment of IR (HOMA-IR), an estimate of IR commonly applied in large epidemiological studies, was used to evaluate IR using the following formula: fasting serum insulin $(\mu \mathrm{U} / \mathrm{mL}) \times$ fasting plasma glucose $(\mathrm{mmol} /$ L)/22.5 [12].

\section{Measures of habitual gait speed and isokinetic leg strength} Habitual gait speed (HGS) was measured in the MEC according to the NHANES standardized protocol. A 20 feet long test tract area was set up in a corridor of the MEC. The 20-foot walk was timed using a hand-held stopwatch. The 20-foot timed walk test was performed at the participant's usual pace. Use of a walker or cane was allowed if needed. HGS was calculated as walking distance $(20$ feet $=$ $6.15 \mathrm{~m}$ ) divided by time (second). Maximal right knee extensor force (Newton) was measured at an angular velocity of 60 degree/second by a Kinetic Communicator isokinetic dynamometer (Chattecx Corp., Chattanooga, $\mathrm{TN})$.

\section{Covariates}

Age, gender, and race/ethnicity were obtained by selfreport. Co-morbidities including heart disease (defined as a history of myocardial infarction, coronary heart disease, congestive heart failure or angina), chronic lung disease (defined as chronic bronchitis or emphysema), and arthritis were ascertained by self-report questionnaires. Alcohol intake was determined by the questionnaire "In any one year, have you had at least 12 drinks of any type of alcohol beverage?" and was dichotomized. Exposure to tobacco smoke increases the concentration of nicotine in the blood, and nicotine is highly specific for such exposure. Cotinine is a major metabolite of nicotine. Using their serum cotinine concentrations $(\mathrm{ng} / \mathrm{mL})$, we classified smoking status of participants in four groups: nonsmoker (<14), light smoker (14-99), moderate smoker (100-199), and heavy smoker $(\geq 200)$ [13]. Three and sometimes 4 blood pressure (BP) determinations were taken using a mercury sphygmomanometer by a NHANES physician. $\mathrm{BP}$ was measured in the right arm unless specific conditions prohibit the use of the right arm. Averaged systolic and diastolic BPs were obtained. The presence of hypertension was defined by a self-report doctor's diagnosis, the use of anti-hypertensive medications, or averaged blood pressure greater than $140 / 90 \mathrm{mmHg}$. Body mass index (BMI), calculated as weight in kilograms divided by the square of height in meters, was categorized according to 
the National Institutes of Health obesity standards: $<18.5$ $=$ underweight, $18.5-24.9=$ normal weight, $25.0-29.9=$ overweight, and $>30=$ obese [14]. C-Reactive protein (CRP) was quantified by utilizing latex-enhanced nephelometry with a Behring Nephelometer Analyzer System (Behring Diagnostics, Frankfurt, Germany). Plasma homocysteine was measured by the Abbott homocysteine assay (Abbott Park, IL, USA), a fully automated fluorescence polarization immunoassay (FPIA) method.

\section{Analysis}

Data were analyzed using Stata 9.0 (Stata, College Station, TX) specialized for complex survey data. We used stratum and primary sampling unit (PSU) variables required for variance estimation. In order to account for clustering and stratification of the multi-stage NHANES sampling design, we incorporated appropriate sampling weight in all analyses in order to obtain point estimates and standard errors (SEs) applicable to the U.S. population. In the NHANES 1999-2002, separate 4-year fasting weights were created to reflect the additional stage of sampling and the additional nonresponse for the subsample of fasting participants assigned to the morning session. These 4-year fasting weights were used for data analysis.

Characteristics of the study population, including means and SEs values for continuous variables and percentages for categorical variables, were calculated with correction for the survey design in both men and women. Stratified by sex, weighted linear regression analysis was used to examine the relation of HOMA-IR to performance-based physical measures, namely leg strength and HGS. The distributions of HOMA-IR in both men and women were right-skewed. Therefore, we used natural-log-transformed values, which provided the best-fitting model for analysis in which the HOMA-IR were treated as a continuous variable. For men, standard-deviation scores of HOMA-IR were obtained from the formula $\left(X_{i}-X_{m}\right) \div S D$, where $X_{i}$ was the natural-log-transformed HOMA-IR in the individual male subject, $\mathrm{X}_{\mathrm{m}}$ the mean natural-log-transformed HOMA-IR in the male subjects, and SD the standard deviation of the natural-log-transformed HOMA-IR in the male subjects. The standard-deviation scores of HOMA-IR in women were obtained from the same formula. This calculation allowed us to determine the change in the gait speed and leg strength for each increment of 1SD in the natural-log-transformed HOMA-IR. The relations of HOMA-IR to leg strength and gait speed were also evaluated with a quartile-based analysis by dividing HOMA-IR levels into quartiles with subjects in the lowest quartile as the reference group. We used an extended-model approach for covariates adjustments: Model $1=$ age, race, BMI, smoking status, alcohol consumption, and use of walking device; Model $2=$ Model $1+$ chronic diseases (hypertension, chronic lung disease, heart diseases, and arthritis); Model 3 = Model $2+$ markers of nutrition and cardiovascular risk (natural-log-transformed levels of folate, vitamin $\mathrm{B}_{12}$, total cholesterol, homocysteine and CRP). Given the fact that muscle strength has been shown to be an important correlate for walking speed among community-dwelling older adults [15], we additionally controlled for leg strength in the association between HOMA-IR and HGS (Model 4) in order to observe possible change of association.

\section{Results}

Table 1 lists the characteristics of the participants according to sex. The mean age among the 566 men was 61.8 years (age range 50 to 85 years), and among the 602 women it was 62.4 years (age range 50 to 85 years). The men had higher fasting plasma glucose, serum insulin, HOMA-IR, peak leg strength, as well as a higher prevalence of heart disease compared to women. The presence of hypertension, chronic lung disease, and arthritis was more common in women than in men. The men smoked more than the women and consumed more alcohol. The cut-off values for HOMA-IR quartiles among the men were: quartile $1(<1.80)$, quartile $2(1.80-2.51)$, quartile 3 (2.52-3.69), and quartile $4(>3.69)$; while among the women they were quartile $1(<1.48)$, quartile $2(1.48$ 2.28 ), quartile 3 (2.29-3.52), and quartile 4 (>3.52). Men were comparable to women in terms of age, BMI, race, and HGS.

HOMA-IR levels were inversely associated with HGS among the men. After adjustment for age, race, BMI categories, alcohol consumption, and use of walking devices, each increment of 1SD in the HOMA-IR level was associated with a $0.035 \mathrm{~m} / \mathrm{sec}$ decrease $(\mathrm{p}=0.007)$ in HGS (Table 2). Additional adjustment of covariates including chronic co-morbidities and markers of nutrition/cardiovascular risk (folate, vitamin B12, total cholesterol, homocysteine, and CRP) did not change the association among men (Model 2 and Model 3). In the full-adjusted model where peak leg strength was additionally adjusted (Model $4)$, the negative association between HOMA-IR and HGS among men remained ( $\beta$ coefficient $-0.042, \mathrm{p}=0.001$ ). We did not find any association between HOMA-IR and HGS among the women. We subsequently divided HOMA-IR levels into quartiles and showed that HGS for men in the highest HOMA-IR quartile were $0.066 \mathrm{~m} / \mathrm{sec}$ less than that for men in the lowest quartile after adjustment for Model 1 covariates (significant trend across HOMA-IR quartiles with $\mathrm{p}=0.035)$. Likewise, supplementary adjustment for additional covariates (Models 2 to Model 4) did not change the inverse association between HOMA-IR and HGS among the men in the quartile-based analyses. We did not find a clear trend between HOMA-IR quartiles and HGS among the women. Stratified by gender, adjusted means of HGS based on different 
Table I: Characteristics of Study Participants NHANES I 999-2002 (N = I | 68)

\begin{tabular}{|c|c|c|c|}
\hline Characteristics & $\begin{array}{c}\text { Men } \\
(N=566)\end{array}$ & Women $(N=602)$ & $P$ value \\
\hline \multicolumn{4}{|l|}{ Continuous variables, Mean (SE) } \\
\hline Age (year) & $61.8(0.4)$ & $62.4(0.3)$ & 0.202 \\
\hline Body mass index $(\mathrm{kg} / \mathrm{m} 2)$ & $27.9(0.3)$ & $27.7(0.3)$ & 0.752 \\
\hline Fasting plasma glucose $(\mathrm{mg} / \mathrm{dL})$ & $101.0(0.5)$ & $97.4(0.5)$ & $<0.001$ \\
\hline Serum insulin $(\mu \mathrm{U} / \mathrm{mL})$ & $11.72(0.4)$ & $10.52(0.2)$ & 0.001 \\
\hline Insulin resistance (HOMA-IR) & $2.96(0.1)$ & $2.59(0.1)$ & $<0.001$ \\
\hline Peak leg strength (Newton) & $439(6.2)$ & $299(4.3)$ & $<0.001$ \\
\hline Habitual gait speed (m/sec) & $1.090(0.013)$ & $1.083(0.011)$ & 0.620 \\
\hline \multicolumn{4}{|c|}{ Categorical variables, $\mathbf{N}$ (weighted \%) } \\
\hline Race & & & 0.427 \\
\hline Mexican American & $104(3.2)$ & $114(3.0)$ & \\
\hline Other Hispanic & $22(3.5)$ & $28(5.2)$ & \\
\hline Non-Hispanic White & $367(85.9)$ & $372(83.5)$ & \\
\hline Non-Hispanic Black & $62(4.8)$ & $77(5.8)$ & \\
\hline All others & II (2.6) & II (2.5) & \\
\hline Hypertension & $294(47.1)$ & $387(58.9)$ & 0.001 \\
\hline Heart diseases & $73(12.4)$ & $50(7.5)$ & 0.017 \\
\hline Chronic lung disease & $30(4.4)$ & $58(I I .4)$ & 0.001 \\
\hline Arthritis & $184(32.5)$ & $278(46.7)$ & $<0.001$ \\
\hline Smoking status & & & 0.001 \\
\hline Nonsmoker & $429(73.3)$ & $514(84.2)$ & \\
\hline Light smoker & $22(3.3)$ & $14(2.3)$ & \\
\hline Moderate smoker & $39(7.3)$ & $35(6.5)$ & \\
\hline Heavy smoker & $76(16.1)$ & $39(7.0)$ & \\
\hline Had > 12 alcohol drinks/year & $464(82.3)$ & $314(60.5)$ & $<0.001$ \\
\hline
\end{tabular}

Values were expressed as mean (SEs) and number (weighted \%)
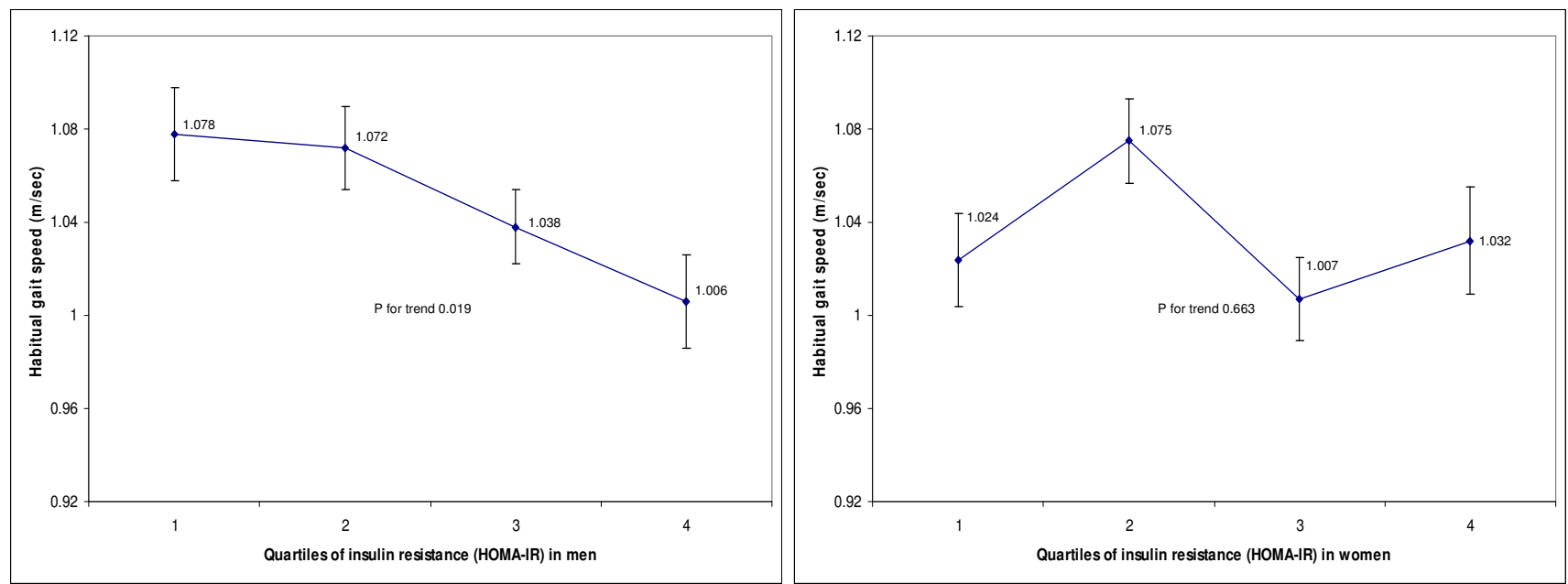

Figure I

Adjusted means of habitual gait speed v.s. quartiles of insulin resistance (HOMA-IR) in both men and women.* * Means of habitual gait speed were adjusted for age, race, body mass index categories, smoking status, alcohol consumption, use of walking devices, co-morbidities (hypertension, chronic lung disease, heart disease, and arthritis), markers of nutrition and cardiovascular risk (natural-log-transformed levels of folate, vitamin $B_{12}$, total cholesterol, homocysteine and C-reactive protein), and peak leg strength. Abbreviation: homeostasis model assessment of insulin resistance HOMA-IR. 
Table 2: Association between insulin resistance (HOMA-IR) and gait speed in men and women

\begin{tabular}{|c|c|c|c|c|}
\hline \multicolumn{5}{|c|}{ Models with HOMA-IR as a continuous variable } \\
\hline \multicolumn{3}{|c|}{ Men } & \multicolumn{2}{|l|}{ Women } \\
\hline & $\begin{array}{c}\beta^{*} \\
\text { (SE) }\end{array}$ & P value & $\begin{array}{c}\beta^{*} \\
\text { (SE) }\end{array}$ & P value \\
\hline Model I & $-0.035(0.013)$ & 0.007 & $-0.018(0.013)$ & 0.172 \\
\hline Model 2 & $-0.033(0.013)$ & 0.010 & $-0.014(0.013)$ & 0.289 \\
\hline Model 3 & $-0.040(0.013)$ & 0.003 & $-0.011(0.013)$ & 0.377 \\
\hline Model 4 & $-0.042(0.012)$ & 0.001 & $-0.012(0.013)$ & 0.364 \\
\hline
\end{tabular}

\begin{tabular}{|c|c|c|c|c|c|c|c|c|}
\hline & \multicolumn{8}{|c|}{ Models with HOMA-IR by increasing quartiles } \\
\hline & \multicolumn{4}{|c|}{ Men } & \multicolumn{4}{|c|}{ Women } \\
\hline & Quartile comparison & $\begin{array}{c}\beta \dagger \\
\text { (SE) }\end{array}$ & P value & $P$ for trend & Quartile comparison & $\begin{array}{c}\beta \dagger \\
\text { (SE) }\end{array}$ & P value & $P$ for trend \\
\hline \multirow[t]{3}{*}{ Model I } & Q2 v.s. QI & $-0.013(0.026)$ & 0.616 & 0.035 & Q2 v.s. QI & $0.049(0.027)$ & 0.072 & 0.446 \\
\hline & Q3 v.s. QI & $-0.043(0.029)$ & 0.141 & & Q3 v.s. QI & $-0.032(0.031)$ & 0.304 & \\
\hline & Q4 v.s. QI & $-0.066(0.033)$ & 0.046 & & Q4 v.s. QI & $-0.001(0.035)$ & 0.987 & \\
\hline \multirow[t]{3}{*}{ Model 2} & Q2 v.s. Q1 & $-0.010(0.026)$ & 0.711 & 0.056 & Q2 v.s. Q1 & $0.052(0.027)$ & 0.057 & 0.521 \\
\hline & Q3 v.s. QI & $-0.039(0.029)$ & 0.177 & & Q3 v.s. QI & $-0.025(0.030)$ & 0.403 & \\
\hline & Q4 v.s. QI & $-0.058(0.033)$ & 0.078 & & Q4 v.s. QI & $0.004(0.033)$ & 0.905 & \\
\hline \multirow[t]{3}{*}{ Model 3} & Q2 v.s. QI & $-0.015(0.027)$ & 0.567 & 0.024 & Q2 v.s. QI & $0.055(0.025)$ & 0.042 & 0.700 \\
\hline & Q3 v.s. QI & $-0.046(0.030)$ & 0.120 & & Q3 v.s. QI & $-0.020(0.030)$ & 0.504 & \\
\hline & Q4 v.s. QI & $-0.072(0.033)$ & 0.030 & & Q4 v.s. QI & $0.013(0.033)$ & 0.702 & \\
\hline \multirow[t]{3}{*}{ Model 4} & Q2 v.s. QI & $-0.006(0.026)$ & 0.786 & 0.019 & Q2 v.s. QI & $0.051(0.026)$ & 0.054 & 0.663 \\
\hline & Q3 v.s. QI & $-0.039(0.028)$ & 0.167 & & Q3 v.s. QI & $-0.018(0.030)$ & 0.556 & \\
\hline & Q4 v.s. QI & $-0.072(0.032)$ & 0.024 & & Q4 v.s. QI & $0.008(0.033)$ & 0.807 & \\
\hline
\end{tabular}

Adjusted covariates:

Model I = Age, race, body mass index categories, smoking status, alcohol consumption, and use of walking devices

Model 2 = Model I + co-morbidities (hypertension, chronic lung disease, heart disease, and arthritis)

Model $3=$ Model $2+$ markers of nutrition and cardiovascular risk (natural-log-transformed levels of folate, vitamin $B_{12}$, total cholesterol,

homocysteine and C-reactive protein).

Model $4=$ Model $3+$ peak leg strength

* Parameter estimates $(\beta)$ can be interpreted as differences in mean gait speed $(\mathrm{m} / \mathrm{sec})$ for each increment of one standard deviation in the log transformed HOMA-IR among men (or women).

† Parameter estimates $(\beta)$ can be interpreted as differences in mean gait speed $(\mathrm{m} / \mathrm{sec})$ compared male (or female) subjects in the $2^{\text {nd }}$, $3^{\text {rd }}$, and $4^{\text {th }}$ quartiles of HOMA-IR to those in the lowest quartile.

Abbreviations: homeostasis model assessment of insulin resistance, HOMA-IR; SE, standard error.

The cut-off values HOMA-IR quartiles among the men were: quartile I $(<1.80)$, quartile $2(I .80-2.5 \mathrm{I})$, quartile $3(2.52-3.69)$, and quartile 4 (>3.69);

while among the women the cut-off values were: quartile I $(<1.48)$, quartile $2(1.48-2.28)$, quartile $3(2.29-3.52)$, and quartile 4 (>3.52).

HOMA-IR quartiles were obtained from the full-adjusted regression models (Model 4) and illustrated in the Figure 1. We did not find any association between HOMA-IR and peak leg strength in both men and women (Data not shown).

\section{Discussion and conclusion}

Among men without diabetes, there was an inverse association between IR and HGS. The relationship was independent of age, race, BMI, smoking status, alcohol consumption, co-morbidities, markers of nutrition and cardiovascular risk, and peak leg strength. Our findings support and extend previous studies examining the role of metabolic syndrome, a cluster of cardiovascular risk factors strongly associated with IR, in predicting the development of functional impairment. Supporting statements from Blazer and colleagues, by analyzing 1229 older adults from the Duke Established Populations for Epidemiologic Studies of the Elderly (EPESE), demonstrated that metabolic syndrome independently predicted mobil- 
ity decline, as assessed by the ability to perform heavy housework unaided, walk up and down a flight of stairs unaided, and walk half a mile unaided[16]. Additionally, the population-based Sacramento Area Latino Study on Aging (SALSA) reinforced the fact that metabolic syndrome was associated with progressive limitations in mobility and strength by following 1606 Mexican Americans older adults for 3 years[17]. Okoro and colleagues, cross-sectionally examining the NHANES cohort, suggested that selected components of metabolic syndrome, specifically low HDL cholesterol and abdominal obesity, were associated with gait impairment[18]. Nevertheless, these studies may have weakness in internal validity because such important confounding factors as chronic inflammation [16-18], medical co-morbidities[16,18], and nutritional status [16-18] were not considered. Furthermore, the reports of functional impairment are selfreported in Blazer et al[16] and Blaum et al[17], thus imposing potential bias in outcome ascertainment. Although metabolic syndrome, a composite outcome defined by expert panel[19], has recently attracted research interest, the usefulness of metabolic syndrome as an actual pathophysiological basis of functional decline has been questioned[20]. On the other hand, IR has been proposed as a principal factor in initiating and perpetuating the pathologic manifestations of the metabolic syndrome[21]. Unfortunately, none of the above studies scrutinize the role of IR in functional status. The crosssectional relation of IR to muscle strength has been evaluated in two population-based studies of community dwellers that highlighted an inverse association between IR and muscle strength [22-24]. However, such studies used different confounding variables in their statistical models and one of the studies consisted of a cohort entirely made up of men[22]which may explain the discrepancies from our findings. To our knowledge, this is the first report to describe the association between IR and performance-based physical function among older men and women by using a large group of geographically dispersed and ethnically diverse national populationbased sample. Potential confounders were comprehensively considered and the role of leg strength in the association between IR and gait speed was assessed.

By acting as a cardiovascular risk factor, IR may cause cerebral atherosclerotic changes in the cerebral circulation [25], thus contributing to the development of cerebral micro-angiopathy (ie, leukoaraiosis or cerebral small vessel disease). The lesions may disrupt the integrity of frontal-subcortical circuits, thus compromising gait function. Cerebrovascular lesions thus provide a conceivable mechanism between IR and slow gait speed. Since the association between IR and gait speed varies across gender, there may be an effect modification of sex in the association. The fact that men had a greater baseline prevalence of cardiovascular risk (such as heart disease and smoking) than women may justify why an inverse association between IR and gait speed is seen in men but not in women. Further research efforts are needed to investigate possible mediating factors, such as differential coordinating processes in brain neural circuiting or sex hormones[26], in mediating the effect of IR on gait speed. Our results have clinical implications. IR, in addition to its metabolic and cardiovascular implications, may serve as an important indicator of walking function among older man. As such, IR may be a promising target for intervention to prevent decline of walking function in late life. Secondly, pharmaceutical interventions such as metformin and thiazolidinedione [27], as well as additional known strategies to effectively lower IR- healthy dietary pattern [28], regular exercise [29], or weight reduction [30]- may additionally improve walking function and prevent functional decline, especially for high-risk subjects.

Our study has potential limitations deserving comments. First, due to the cross-sectional design, causal relationship between IR and gait speed can not be established. The association between IR and gait speed could simply be epiphenomenon of aging and the association should be prospectively explored. Second, although we have comprehensively adjusted for such confounders as chronic comorbidities, markers of nutrition, inflammation, and cardiovascular risk, as well as leg strength in the association of IR and gait speed, other important measures such as cognitive ability, symptoms of depression, markers of endothelial dysfunction, or evidence of cerebral small vessel disease are absent or incomplete in the NHANES 19992002 dataset.

In conclusion, a higher level of IR was associated with a slower gait speed among non-diabetic older men. The association does not exist in the women. We provided new information for the association between IR and gait speed among community-dwelling older adults where data currently do not exist.

\section{Competing interests}

The authors declare that they have no competing interests.

\section{Authors' contributions}

All authors critically reviewed the manuscript, read and approved the final manuscript.

\section{Acknowledgements}

The study was sponsored by the National Health and Research Institutes, Taipei, Taiwan.

\section{References}

I. Abbatecola AM, Paolisso G, Lamponi M, Bandinelli S, Lauretani F, Launer L, Ferrucci L: Insulin resistance and executive dysfunc- 
tion in older persons. Journal of the American Geriatrics Society 2004, 52(10): $17|3-17| 8$.

2. Geroldi C, Frisoni GB, Paolisso G, Bandinelli S, Lamponi M, Abbatecola AM, Zanetti $O$, Guralnik JM, Ferrucci L: Insulin resistance in cognitive impairment: the InCHIANTI study. Archives of neurology 2005, 62(7): 1067-1072.

3. Barzilay JI, Blaum C, Moore T, Xue QL, Hirsch CH, Walston JD, Fried LP: Insulin resistance and inflammation as precursors of frailty: the Cardiovascular Health Study. Archives of internal medicine 2007, 167(7):635-64I.

4. Manton KG, Corder LS, Stallard E: Estimates of change in chronic disability and institutional incidence and prevalence rates in the U.S. elderly population from the 1984, and 1989 National Long Term Care Survey. J Gerontol I982, 48(4):SI53-166.

5. Guralnik JM, Ferrucci L, Simonsick EM, Salive ME, Wallace RB: Lower-extremity function in persons over the age of 70 years as a predictor of subsequent disability. N Engl J Med 1995, 332(9):556-56I.

6. Ostir GV, Markides KS, Black SA, Goodwin JS: Lower body functioning as a predictor of subsequent disability among older Mexican Americans. J Gerontol A Biol Sci Med Sci 1998, 53(6):M49|-495.

7. Giampaoli S, Ferrucci L, Cecchi F, Lo Noce C, Poce A, Dima F, Santaquilani A, Vescio MF, Menotti A: Hand-grip strength predicts incident disability in non-disabled older men. Age Ageing 1999 , 28(3):283-288.

8. Shinkai S, Watanabe S, Kumagai S, Fujiwara $Y$, Amano $\mathrm{H}$, Yoshida $\mathrm{H}$, Ishizaki T, Yukawa H, Suzuki T, Shibata $\mathrm{H}$ : Walking speed as a good predictor for the onset of functional dependence in a Japanese rural community population. Age Ageing 2000, 29(5):44I-446.

9. Rantanen T, Avlund K, Suominen H, Schroll M, Frandin K, Pertti E: Muscle strength as a predictor of onset of ADL dependence in people aged 75 years. Aging Clin Exp Res 2002, I 4(3 Suppl): $10-15$.

10. 1999-2000 National Health and Nutrition Examination Survey (NHANES) [http://www.cdc.gov/nchs/nhanes.htm]

II. 200 I-2002 National Health and Nutrition Examination Survey (NHANES) [Http://www.cdc.gov/nchs/about/major/nhanes/]. Bathesda, MD: National Center for Health Statistics

12. Matthews DR, Hosker JP, Rudenski AS, Naylor BA, Treacher DF, Turner RC: Homeostasis model assessment: insulin resistance and beta-cell function from fasting plasma glucose and insulin concentrations in man. Diabetologia 1985, 28(7):412-419.

13. Wei W, Kim Y, Boudreau N: Association of smoking with serum and dietary levels of antioxidants in adults: NHANES III, 1988-1 994. American journal of public health 200 I, 9 I (2):258-264.

14. National Heart L, and Blood Institute: Obesity Education Initiative: The Evidence Report Bethesda, MD: Nation Institutes of Health; 1998.

15. Ostchega Y, Dillon CF, Lindle R, Carroll M, Hurley BF: Isokinetic leg muscle strength in older americans and its relationship to a standardized walk test: data from the national health and nutrition examination survey 1999-2000. Journal of the American Geriatrics Society 2004, 52(6):977-982.

16. Blazer DG, Hybels CF, Fillenbaum GG: Metabolic syndrome predicts mobility decline in a community-based sample of older adults. Journal of the American Geriatrics Society 2006, 54(3):502-506.

17. Blaum CS, West NA, Haan MN: Is the metabolic syndrome, with or without diabetes, associated with progressive disability in older Mexican Americans? J Gerontol A Biol Sci Med Sci 2007, 62(7):766-773.

18. Okoro CA, Zhong Y, Ford ES, Balluz LS, Strine TW, Mokdad AH: Association between the metabolic syndrome and its components and gait speed among U.S. adults aged 50 years and older: a cross-sectional analysis. BMC Public Health 2006, 6:282

19. Executive Summary of The Third Report of The National Cholesterol Education Program (NCEP) Expert Panel on Detection, Evaluation, And Treatment of High Blood Cholesterol In Adults (Adult Treatment Panel III). JAMA 200I, 285(19):2486-2497.

20. Kahn R, Buse J, Ferrannini E, Stern M: The metabolic syndrome: time for a critical appraisal: joint statement from the American Diabetes Association and the European Association for the Study of Diabetes. Diabetes Care 2005, 28(9):2289-2304.
21. Lann D, LeRoith D: Insulin resistance as the underlying cause for the metabolic syndrome. Med Clin North Am 2007, 9I(6): 1063-1077.

22. Lazarus R, Sparrow D, Weiss ST: Handgrip strength and insulin levels: cross-sectional and prospective associations in the Normative Aging Study. Metabolism 1997, 46(I I): I266-1269.

23. Abbatecola AM, Ferrucci L, Ceda G, Russo CR, Lauretani F, Bandinelli S, Barbieri M, Valenti G, Paolisso G: Insulin resistance and muscle strength in older persons. J Gerontol A Biol Sci Med Sci 2005, 60(10): $1278-1282$.

24. Barzilay JI, Cotsonis GA, Walston J, Schwartz AV, Satterfield S, Miljkovic I, Harris TB: Insulin resistance is associated with decreased quadriceps muscle strength in nondiabetic adults aged >or $=70$ years. Diabetes Care 2009, 32(4):736-738.

25. Bertoni AG, Wong ND, Shea S, Ma S, Liu K, Preethi S, Jacobs DR Jr. Wu C, Saad MF, Szklo M: Insulin resistance, metabolic syndrome, and subclinical atherosclerosis: the Multi-Ethnic Study of Atherosclerosis (MESA). Diabetes Care 2007, 30(I i):295I-2956.

26. Morrison JH, Brinton RD, Schmidt PJ, Gore AC: Estrogen, menopause, and the aging brain: how basic neuroscience can inform hormone therapy in women. J Neurosci 2006, 26(4I): $10332-10348$

27. Staels B: Metformin and pioglitazone: Effectively treating insulin resistance. Curr Med Res Opin 2006, 22(Suppl 2):S27-37.

28. Esmaillzadeh A, Kimiagar M, Mehrabi Y, Azadbakht L, Hu FB, Willett WC: Dietary patterns, insulin resistance, and prevalence of the metabolic syndrome in women. Am J Clin Nutr 2007, 85(3):910-918.

29. Hawley JA: Exercise as a therapeutic intervention for the prevention and treatment of insulin resistance. Diabetes/metabolism research and reviews 2004, 20(5):383-393.

30. Mazzali G, Di Francesco V, Zoico E, Fantin F, Zamboni G, Benati C, Bambara V, Negri M, Bosello O, Zamboni M: Interrelations between fat distribution, muscle lipid content, adipocytokines, and insulin resistance: effect of moderate weight loss in older women. Am J Clin Nutr 2006, 84(5): I I93-I I 99.

\section{Pre-publication history}

The pre-publication history for this paper can be accessed here:

http://www.biomedcentral.com/1471-2318/9/49/prepub

Publish with BioMed Central and every scientist can read your work free of charge

"BioMed Central will be the most significant development for disseminating the results of biomedical research in our lifetime. "

Sir Paul Nurse, Cancer Research UK

Your research papers will be:

- available free of charge to the entire biomedical community

- peer reviewed and published immediately upon acceptance

- cited in PubMed and archived on PubMed Central

- yours - you keep the copyright 